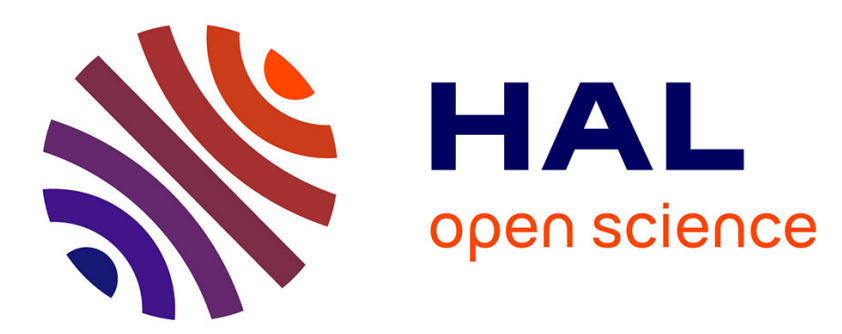

\title{
A simple and versatile method for statistical analysis of the electrical properties of individual double walled carbon nanotubes
}

Florent Seichepine, Emmanuel Flahaut, Christophe Vieu

\section{- To cite this version:}

Florent Seichepine, Emmanuel Flahaut, Christophe Vieu. A simple and versatile method for statistical analysis of the electrical properties of individual double walled carbon nanotubes. Microelectronic Engineering, 2011, 88 (7), pp. 1637-1639. 10.1016/j.mee.2011.01.081 . hal-00828791

\section{HAL Id: hal-00828791 https://hal.science/hal-00828791}

Submitted on 31 May 2013

HAL is a multi-disciplinary open access archive for the deposit and dissemination of scientific research documents, whether they are published or not. The documents may come from teaching and research institutions in France or abroad, or from public or private research centers.
L'archive ouverte pluridisciplinaire HAL, est destinée au dépôt et à la diffusion de documents scientifiques de niveau recherche, publiés ou non, émanant des établissements d'enseignement et de recherche français ou étrangers, des laboratoires publics ou privés. 


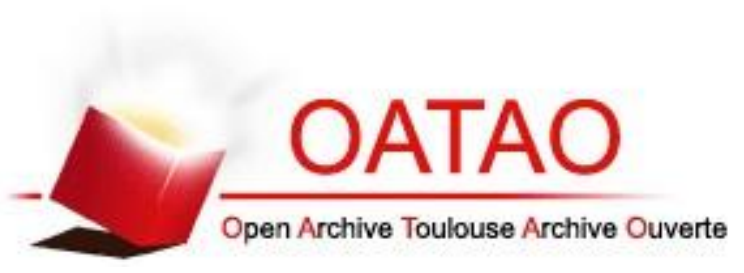

\section{Open Archive Toulouse Archive Ouverte (OATAO)}

OATAO is an open access repository that collects the work of Toulouse researchers and makes it freely available over the web where possible.

This is an author-deposited version published in: http://oatao.univ-toulouse.fr/ Eprints ID: 8692

To link to this article: DOI:10.1016/j.mee.2011.01.081

Official URL: http://dx.doi.org/10.1016/j.mee.2011.01.081

\section{To cite this version:}

Seichepine, Florent and Flahaut, Emmanuel and Vieu, Christophe A simple and versatile method for statistical analysis of the electrical properties of individual double walled carbon nanotubes. (2011) Microelectronic Engineering, vol. 88 (n ○ 7). pp. 1637-1639. ISSN 0167-9317

Any correspondence concerning this service should be sent to the repository administrator: staff-oatao@inp-toulouse.fr 


\title{
A simple and versatile method for statistical analysis of the electrical properties of individual double walled carbon nanotubes
}

\author{
Florent Seichepine ${ }^{\mathrm{a}, \mathrm{b}, \mathrm{c}, \mathrm{d}, *}$, Emmanuel Flahaut ${ }^{\mathrm{a}, \mathrm{c}}$, Christophe Vieu $^{\mathrm{b}, \mathrm{d}}$ \\ a Université de Toulouse; UPS, INP; Institut Carnot Cirimat; 118, route de Narbonne, F-31062 Toulouse, cedex 9, France \\ ${ }^{\mathrm{b}}$ Université de Toulouse, UPS, INSA, INP, ISAE, LAAS F-31059, France \\ ${ }^{\mathrm{c}}$ CNRS, Institut Carnot Cirimat, F-31062 Toulouse, France \\ ${ }^{\mathrm{d}}$ CNRS-LAAS, 7avenue du colonel Roche, F-31077 Toulouse, France
}

Keywords:

\begin{abstract}
A B S T R A C T
Double-walled carbon nanotubes (DWNTs) are potential candidates for new generation of on chip interconnections due to their nearly metallic behaviour. For such large scale integration purpose it is mandatory to characterize their electrical properties in a statistical way. We thus propose a new methodology for characterizing in one step, the electrical properties of a large population of nanotubes. The method enables to obtain histograms of the conductance and maximum current density of individual nanoobjects.
\end{abstract}

\section{Introduction}

The use of metallic carbon nanotubes bundles is investigated as an alternative to copper for the next generation of on chip interconnections. CNT bundles can outperform copper interconnects in terms of propagation delay for local, intermediate and global wires [1]. The other advantage of those nano-objects in the microelectronics field is their ability to carry extremely high current densities $\left(>10^{9}\left[\mathrm{~A} \mathrm{~cm}^{2}\right]\right.$ ] [2] (about 100 times more than copper). The realisation of this new kind of CNT-based interconnections also requires major innovations in the field of large scale processing. The challenge is to find an efficient way to integrate dense bundles of oriented CNTs at wafer scale. Several techniques are currently investigated [3-7]. One of the problems to obtain devices with controlled behaviours is the large dispersion of conduction properties observed in CNT samples. The number of walls, the chirality, the defect concentration, the surface properties, the length and the diameter are generally different from one tube to another. Those characteristics are leading to very different conduction behaviour which can be detrimental for VLSI applications. To alleviate this problem one of the possibility relies on CNTs sorting in order to reduce the dispersion of the conduction properties of the initial mate-

\footnotetext{
* Corresponding author at: Université de Toulouse, UPS, INP, Institut Carnot Cirimat; 118, route de Narbonne, F-31062 Toulouse, cedex 9, France.

E-mail address: seichepine@gmail.com (F. Seichepine).
}

rial [8]. The control of the properties may also be achieved by using specific catalysts to obtain only one desired CNT type [9]. In any case, it seems very important to develop a reliable method in order to statistically measure the conduction properties of a given batch of CNTs. Such a method could be essential for investigating the most suitable process for tuning the metallic properties of CNTs and for qualifying any CNT material for on chip interconnects.

This paper describes a new methodology for one-step characterization of the electrical properties of a large population of carbon nanotubes. This original method relies on the parallel integration of many CNTs on a set of microelectrodes and the careful investigation of their electrical properties. The method enables to obtain histograms of the electrical conductivity and maximum current density of individual nano-objects.

\section{Microelectrode fabrication}

Metallic microelectrodes were fabricated on a 4 inch silicon wafer. A thermal oxide layer ( $300 \mathrm{~nm}$ ) was grown to avoid any conduction through the substrate and UV proximity photolithography using AZ1529 photoresist was implemented for generating the patterns. The metal electrodes were obtained by a lift-off process of a thin metallic film composed of Ti and $\mathrm{Au}\left(\mathrm{Ti}_{10 \mathrm{~nm}}\right.$ $\left(\mathrm{Au}_{100 \mathrm{~nm}}\right)$. Gold electrodes are known to decrease the contact resistance with CNTs [10]. A large number of electrodes were designed on the wafer (about 19,000). The electrodes are $125 \mu \mathrm{m}$ long, 

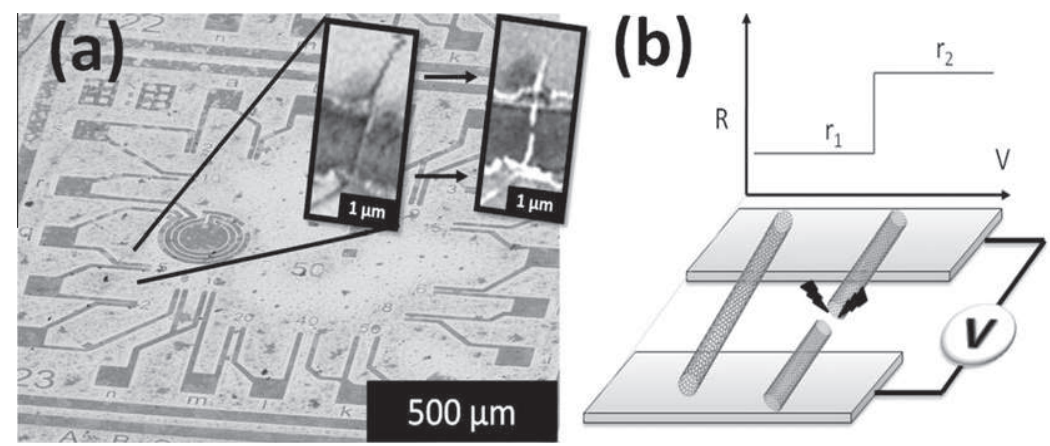

Fig. 1. (a) SEM image of one of the test chip. In the inset, a CNT before and after the breakdown. (b) Schematic view of the principle of our experiments.

$10 \mu \mathrm{m}$ wide and exhibit insulating gaps ranging from 1 to $50 \mu \mathrm{m}$. Different orientations and shapes of electrodes are designed on the substrate (Fig. 1 is a SEM image of a typical one).

\section{CNT synthesis and deposition}

DWNTs used in this work are prepared in-house by Catalytic Chemical Vapour Deposition (CCVD), by decomposition of $\mathrm{CH}_{4}$ at $1000{ }^{\circ} \mathrm{C}\left(\mathrm{H}_{2}: \mathrm{CH}_{4} \mathrm{~atm}\right.$. $)$ [11]. After catalyst removal $(\mathrm{HCl})$, the sample contains approximately $80 \%$ of DWNTs, the rest being mainly SWNTs ( 15\%) and Triple-Walled Nanotubes. The large proportion of metallic CNTs obtained in these conditions represents a huge advantage for interconnect applications [12]. The DWNTs were then purified by air oxidation $\left(500^{\circ} \mathrm{C}, 30 \mathrm{~min}\right)$ to finally obtain high-purity samples [13]. A suspension in ethanol was prepared by mixing the obtained DWNT powder with high purity ethanol using a sonication probe. Suspensions of different concentrations have been prepared. The suspensions were then sprayed using a manual airbrush. The use of a spray coating technique allows a very homogeneous deposition of CNTs over the entire substrate. By controlling the concentration of the solution and the deposition parameters, we could adjust the CNT density on the substrate.

\section{Electrical characterization}

The aim of this study was to find an efficient way for testing simultaneously the electrical properties of large assemblies of CNTs. This is required when one wishes to investigate the influence of synthesis conditions or chemical treatments applied to a CNT sample. The main parameters of interest for interconnects applications are the maximum current density and the resistivity of CNT wires. By controlling the CNT concentration in the ethanol suspension and the deposition parameters, we could obtain electrodes connected with only few CNTs (between 1 and 10). The inset in the Fig. 1a is a SEM picture of such a connection (after breakdown). A disrupted CNT bundle is visible between the gold electrodes.

The electrical tests have been performed using an Agilent 4142B Modular DC Source/Monitor used with a Suss Microtec PA200 tip station. The experiment was driven by Metrics ICS software. The temperature of the substrate was carefully maintained at $25^{\circ} \mathrm{C}$ using the tip station and the tests have been performed under ambient atmosphere. Depending on the length of the nano-object tested, an adapted gap is chosen. In our case, $5 \mu \mathrm{m}$ gaps between electrodes were appropriate for connecting efficiently the devices.The electrical characteristics were obtained by sweeping the voltage from 0 to $60 \mathrm{~V}$ and measuring the current flowing through the CNTs bridging the gaps of the microelectrodes. However, in order to decrease the resistance contact and avoid the influence of adsorbed species on CNTs, a heat treatment was systematically performed before each measurement. A sweep of voltage from 0 to $4 \mathrm{~V}$ in about $5 \mathrm{~s}$ was applied and repeated four times, which induced a heating by joule effect on the CNTmetal contacts. This treatment allowed us to obtain more linear $I(V)$ characteristics [14]. A typical $I(V)$ curve is shown in Fig. 2. The voltage sweep between the two electrodes leads to the successive destruction of individual CNT [15]. Even if this operation is performed simultaneously on several CNTs, it is however possible to extract the electrical properties of single nano objects thanks to the data analysis proposed and detailed below. The sequential breakdown of CNTs leads to a progressive increase in the global resistance of the system. This setup can be used to characterise very rapidly a large amount of individual CNTs on a large number of connections.

\section{Data analysis}

The $I(V)$ traces exhibit several very precise steps (Fig. 2). As a consequence, the $R(V)$ curves present a step by step increase in the resistance as the voltage increases. The values of the resistance registered on the plateaux are very stable. The hypothesis is made that the sharp resistance increase corresponds to the destruction of a single conduction path. SEM observations of such connections, before and after the electrical stimulation, support this hypothesis (see inset of the Fig 1a). It has been reported, that breakdown occurred when the temperature reaches about $600^{\circ} \mathrm{C}$ on the CNT. In our case, due to the low thermal conductivity of silicon dioxide compared to the gold electrode, the maximal temperature is probably localised at the centre of the CNT [16]. The final value of the resistance corresponds to the resistance level of an unconnected microelectrode gap. We have also verified many times that the number of steps, observed in the $I / V$ curve, corresponds to the number of CNTs which could be observed by SEM.

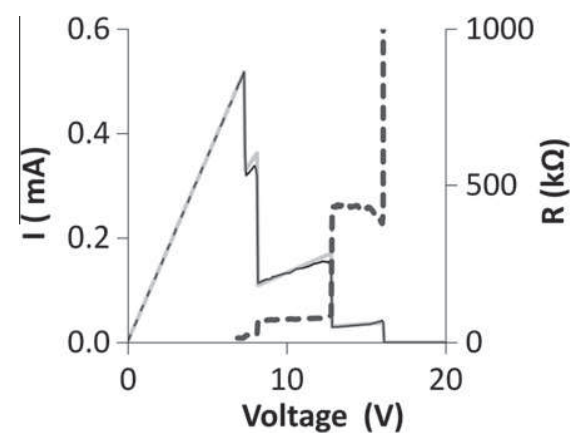

Fig. 2. Typical examples of the $I(V)$ (black line) and $R(V)$ (dashed line) curves obtained by our protocol . The $I(V)$ is compared with the calculated model (grey line). 


\section{Calculations}

To find out the electrical parameters of single conduction pathways, data processing was necessary. For such a purpose, we approximate our system as a range of perfect resistances arranged in parallel. Some valuable parameters can be directly extracted from our measurements. Each local maximum gives the $I_{\max , \text { tot }}$ and the $V_{\text {max,tot }}$ which are respectively the current and the voltage between the electrodes, just before a conduction pathways is disrupted. We can also calculate the global resistance of the system $\left(R_{\text {tot }}\right)$. By using those measurements, we are able to isolate the properties of each conduction pathways. First, we define $R_{1}, R_{2}$, $\ldots, R_{n}$ as the resistance of each conduction pathways. We can then define $R_{\mathrm{tot}}$ as follows:

$\operatorname{Rtot}(V)=\frac{1}{\frac{1}{R 1}+\frac{1}{R 2}+\frac{1}{R 3}+\frac{1}{R 4}+\ldots+\frac{1}{R n}}$

where $n$ is the total number of conduction pathways. $R_{\text {tot }}$ is increasing step by step during the voltage sweep. In fact, one after another, conduction pathways are destroyed and the Ri (with $i=n$ to 1 ) factors disappears. $R_{\text {tot }}$ can be described as a series of constants. We define $R a, R b$ and so on, by

$R a=\frac{1}{\frac{1}{R 1}+\frac{1}{R 2}+\frac{1}{R 3}+\frac{1}{R 4}+\ldots+\frac{1}{R n}} ; R b=\frac{1}{\frac{1}{R 1}+\frac{1}{R 2}+\frac{1}{R 3}+\frac{1}{R 4}+\ldots+\frac{1}{R n-1}}$

Then we can calculate each factor by the following equation:

$\frac{1}{R a}-\frac{1}{R b}=\frac{1}{R 1}+\ldots+\frac{1}{R n}-\left(\frac{1}{R 1}+\ldots+\frac{1}{R n-1}\right)=\frac{1}{R n}$.

With the same calculation repeated $n$ times, we can obtain the individual resistance of each conduction path. Finally, through the simple relation $V_{\text {max, tot }}=R_{i} \times I_{\text {max, tot }}$ for each resistance, we can calculate the maximum current $I_{\max }$ supported by each path.

The average diameter of the CNTs or CNT bundle has been measured by AFM. We assumed that the effective length of the CNTs is the length of the gap between the electrodes regardless of their orientation. Then the maximum current density and the resistivity of each conduction pathways could be calculated. It must be pointed out however, that those figures do not correspond to the intrinsic properties of individual CNT, but rather describe the CNTs with their environment at the metal/CNT interface. This is not a limitation because for interconnect applications, these contacts will be also present. Moreover, the use of an average diameter for all the connections allows fast computing, but induces a quite large approximation when the distribution is large. To validate our hypothesis we simulated the response to a voltage sweep of a system of resistances using our extracted values. The comparison between the measured data and the response of the equivalent circuit gives us an indication on the validity of our calculations and hypothesis. An example can be seen in Fig. 1b (comparison between the black (measurement) and grey (equivalent circuit) plots.

This protocol has been used to test several connections with a semi automatic procedure. This technique is very efficient for electrodes connected by one to about twenty CNTs. The systematic comparison between the original $I(V)$ data and the corresponding simulated $I(V)$ curve show that our assumptions and model describes the experiment with an accuracy better than 5\%. Finally, histograms of individual electrical characteristics can be extracted. Fig. 3 shows, as an example, the distribution of maximum current density capable to flow through DWNTs which have been previously chemically oxidized using $\mathrm{HNO}_{3}$. In this case, we can see a quite large distribution of maximum current density, with an aver-

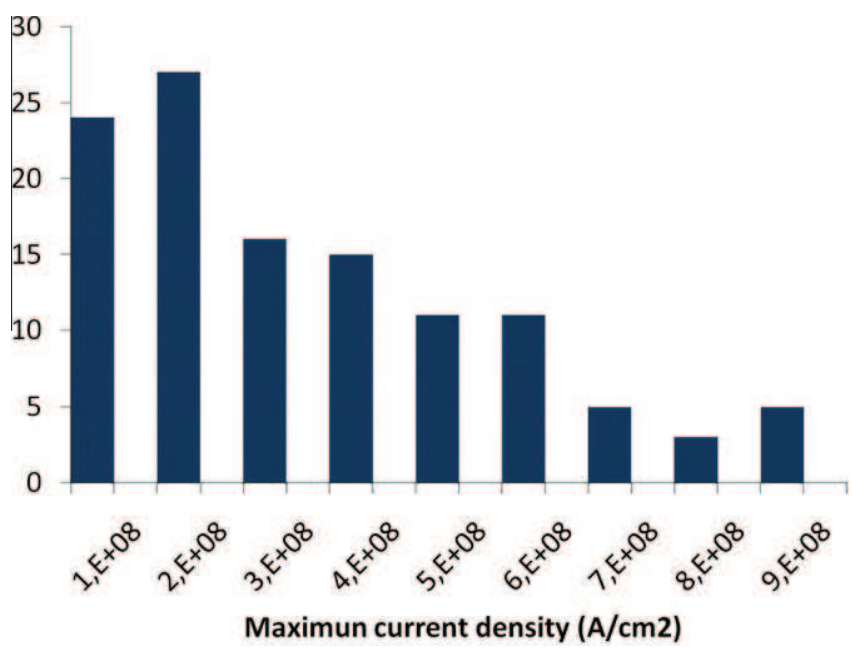

Fig. 3. Example of an histogram of maximum current densities measured on a dispersion of $\mathrm{HNO}_{3}$ oxidized DWNTs.

age value below previous reports of the literature. This observation can be explained by the possible damage that occurred on the CNTs structure during the oxidation process.

\section{Conclusion and perspectives}

We have proposed an electrical method for measuring with high throughput the electrical characteristics of any CNT suspension. Without any advanced lithography steps we can record massive measurements of individual nanotubes. The method enables to obtain clean statistical data over a large population of CNTs and permits a much more accurate comparison between different populations of CNTs than previous proposals. Indeed rather than comparing average values as usually done, we are able to compare histograms of electrical parameters.

\section{References}

[1] A. Naeemi, R. Sarvari, J.D. Meindl, Electron Device Letters, IEEE 84 (2005).

[2] B. Wei, R. Spolenak, P. Kohler-Redlich, M. Ruhle, E. Arzt, Applied Physics Letters 74 (1999) 3149.

[3] Q. Cao, J.A. Rogers, Nano Research 1 (2008) 259.

[4] L.A. Nagahara, I. Amlani, J. Lewenstein, R.K. Tsui, Applied Physics Letters 80 (2002) 3826.

[5] S. Lyonnais, L. Goux-Capes, C. Escudï ¿ㅉㄹㄹ D. Cote, A. Filoramo, J.-P. Bourgoin, Small 4 (2004) 442.

[6] M. Hiramatsu, T. Deguchi, H. Nagao, M. Hori, Japanese Journal of Applied Physics 46 (2007) L303.

[7] J.C. Coiffic, M. Fayolle, P. Faucherand, M. Levis, H.L. Poche, J. Dijon, S. Maitrejean, Physica Status Solidi (a) 205 (2008) 1399.

[8] M.C. Hersam, Nature Nanotechnology 3 (2008) 387.

[9] R. Jasti, J. Bhattacharjee, J.B. Neaton, C.R. Bertozzi, Journal of the American Chemical Society 130 (2008) 17646.

[10] G. Close, H.-S. Wong, Nanotechnology IEEE Transactions 7 (2008) 596.

[11] E. Flahaut, R. Bacsa, A. Peigney, C. Laurent, Chemical Communications (2003) 1442.

[12] P. Puech, A. Ghandour, A. Sapelkin, C. Tinguely, E. Flahaut, D.J. Dunstan, W. Bacsa, Physical Review B 78 (2008) 045413.

[13] S. Osswald, E. Flahaut, H. Ye, Y. Gogotsi, Chemical Physics Letters 402 (2005) 422.

[14] L. Dong, S. Youkey, J. Bush, J. Jiao, V.M. Dubin, R.V. Chebiam, Journal of Applied Physics 101 (2007) 024320.

[15] A. Javey, J. Guo, M. Paulsson, Q. Wang, D. Mann, M. Lundstrom, H. Dai, Physical Review Letters 92 (2004) 106804.

[16] E. Pop, Nanotechnology 1 (2008) 295202. 